\title{
MiR-381 inhibits migration and invasion in human gastric carcinoma through downregulatedting SOX4
}

\author{
MINGMING ZHANG ${ }^{1}$, SHISHU HUANG ${ }^{2}$ and DAN LONG ${ }^{3}$ \\ ${ }^{1}$ Department of Gastroenterological Surgery; ${ }^{2}$ Department of Orthopaedic Surgery; ${ }^{3}$ Key Laboratory of Transplant \\ Engineering and Immunology, West China Hospital, Sichuan University, Chengdu, Sichuan 610041, P.R. China
}

Received January 26, 2017; Accepted June 2, 2017

DOI: $10.3892 / 01.2017 .6637$

\begin{abstract}
Aberrant expression of microRNAs (miRs) serves essential roles in the generation and progression of various types of human cancer. In the present study, the expression and biological functions of miR-381 in human gastric carcinoma (GC) were focused upon. The results of reverse transcription-quantitative polymerase chain reaction analysis revealed that the expression of miR-381 was significantly downregulated in GC tissue samples. Furthermore, low expression of miR-381 was identified to be associated with lymphatic metastasis and advanced tumor-node-metastasis stage (III+IV). Upregulation of miR-381 inhibited the migration and invasion of GC SGC-7901 cells through SRY-Box 4 (SOX4)-mediated epithelial-mesenchymal transition. Finally, long non-coding (lnc) RNA-taurine upregulatedted 1 (non-protein coding) (TUG1) was confirmed as a negatively regulator of miR-381 expression in SGC-7901 cells. Taken together, the results of the current study indicate that the downregulation of miR-381 by IncRNA-TUG1 promoted the metastasis of GC cells by inhibiting SOX4. Thus, targeting miR-381 may be a novel therapeutic option for the treatment of patients with GC.
\end{abstract}

\section{Introduction}

Gastric carcinoma (GC) is one of the most common types of gastrointestinal cancers (1). Local and systemic metastasis mainly decided its poor prognosis (2). At present, identifying the potential molecular target of the metastatic process is critical for the diagnosis and treatment of gastric carcinoma.

MicroRNAs (miRNAs) are a subset of noncoding transcripts shorter than 25 nucleotides (3). Despite of their short length, miRNAs have been confirmed to act vital important

Correspondence to: Dr Mingming Zhang, Department of Gastroenterological Surgery, West China Hospital, Sichuan University, 37 Guo Xuan Xiang, Chengdu, Sichuan 610041, P.R. China

E-mail: huaxizhangmingming@126.com

Key words: miR-381, migration, invasion, SOX4, gastric carcinoma roles in various biological processes including glycometabolism (4), angiogenesis (5), cell growth (6) and movement (7).

MicroRNA-381 (miR-381) was shown to serve as a tumor suppressor in breast cancer (8), chondrosarcoma (9), osteosarcoma (10) and ovarian cancer (11). In these cancers, the expression levels of miR-381 were commonly downregulated; over-expression of miR-381 in vitro could play anti-cancer roles by growth arrest and metastasis inhibition through targeting Cx43, VEGF-C, LRRC4 and YY1. However, the expression and functions of miR-381 in gastric carcinoma are still unclear. Recently, long noncoding RNAs (lncRNAs) were found to act as a sponge of microRNAs and regulate the expression and function of microRNAs (12), but there is still no study to investigate whether miR-381 can be regulated by IncRNAs.

In this study, we revealed that the expression of miR-381 was significantly downregulatedted in GC tissues, especially in metastatic patients. Functionally, we found that over-expression of miR-381 could inhibit the metastatic ability including migration and invasion, as well as epithelial-mesenchymal transition (EMT) of GC cells through targeting SOX4. Furthermore, the present study elucidated that long noncoding RNA TUG1 was a direct negative regulator of miR-381 expression in gastric carcinoma.

\section{Materials and methods}

Patients and cell culture. All protocols were approved by the Huaxi Hospital Ethics Committee (Chengdu, Sichuan, China). Gastric carcinoma tissues and matched tumor-adjacent tissues were collected from $60 \mathrm{GC}$ patients including 36 males and 24 females who received surgical resection between April 2013 and June 2015. The age range was between 45 and 73 years, and the median age was 54 years.

GES-1, BGC-823, MGC-803, SGC-7901 and MKN28 cells were bought from American Type Culture Collection and cultured in Dulbecco's modified Eagle's medium (DMEM; Invitrogen; Waltham, MA, USA) containing 10\% fetal bovine serum (FBS, Gibco, Grand Island, NY, USA), $1 \%$ penicillin and $1 \%$ streptomycin.

Real time quantitative reverse transcription-PCR (qRT-PCR). TRIzol (Life Technologies, Grand Island, NY, USA) regant was used to isolate total RNA from tissues and cells according 
to the instructions. To determine the expression levels of miR-381, SOX4 mRNA and lncRNA-TUG1, a quantitative one-step Perfect Real Time RT-qPCR (SYBR-Green I) kit (Takara Biotechnology Co., Ltd., Dalian, China) was used to synthetize cDNA and amplify target genes. MiR-381 and RNU6 Bulge-Loop ${ }^{\mathrm{TM}}$ primers were purchased from RiboBio Co., Ltd. (Guangzhou, China). SOX4, IncRNA-TUG1 and GAPDH primers were synthetized by Sangon Co., Ltd. (Shanghai, China). Sequences of these primers were shown as follow. SOX4 primers: 5'-AGCGACAAGATCCCTTTC ATTC-3' (forward) and 5'-CGTTGCCGGACTTCACCTT-3' (reverse); IncRNA-TUG1: 5'-TAGCAGTTCCCCAATCCT TG3-3' (forward) and 5'-CACAAATTCCCATCATTCCC-3' (reverse); GAPDH: 5'-CGGAGTCAACGGATTTGGTCG TAT-3' (forward) and 5'-AGCCTTCTCCATGGTGGTGAA GAC-3' (reverse). GAPDH was used as an endogenous control for SOX4 and lncRNA-TUG1. RNU6 served as an endogenous control for miR-381.

Cell transfection. The miR-381 mimics, negative control mimics (miR-NC), siRNAs against lncRNA-TUG1 (forward: 5'-CCCUCCAUGAAUACCUGAATT-3', reverse: 5'-UUC AGGUAUUCAUGGAGGGTT-3') and the negative control siRNA (si-NC) were bought from RiboBio Co., Ltd. These mimics and siRNAs mentioned above were transfected into GC cells with Lipofectamine 3000 according to the manufacturer's instructions (Invitrogen, Waltham, MA, USA).

Wound healing assay. SGC-7901 cells were transfected with miR-381 mimics or NC mimics for $24 \mathrm{~h}$. Cells were seeded onto six-well plate until the fusion reached above 90\%. $100 \mu \mathrm{l}$ tip was used to make a wound at the middle of the well. Remnant cells were washed away by PBS. Serum-free DMEM medium was used to culture cells for $48 \mathrm{~h}$. The healing of the wound was observed under the inverted microscope.

Transwell assay. GC cells were re-suspended with serum-free DMEM medium at a concentration of $2.5 \times 10^{5} / \mathrm{ml} .200 \mu \mathrm{l}$ cells were seeded onto the upper well of Matrigel-coated (BD Biosciences, Franklin Lakes, NJ, USA) $8-\mu \mathrm{m}$ pore Transwell inserts (Nalge Nunc International, Penfield, NY, USA) for invasion assay or uncoated transwell inserts for migration assay. $750 \mu \mathrm{l}$ DMEM medium with $10 \%$ FBS was added to lower chamber. Cells were cultured $24 \mathrm{~h}$ for invasion assay or $48 \mathrm{~h}$ for migration assay. After that, cells in the lower surface were stained with $0.1 \%$ crystal violet, and cell numbers were counted from 10 random fields of the lower surface of the filter.

IHC staining. SP link IHC Detection Kits (Biotin-Streptavidin HRP Detection Systems, SP-9001, including goat-rabbit secondary antibodies) was purchased from Zhongshan Golden Bridge Biotechnology, Inc. (Beijing, China). In brief, rabbit anti-human polyclonal SOX4 (ab80261, dilution, 1:100) antibodies were purchased from Abcam Biotechnology, Inc. (Cambridge, MA, USA) and used to detect the protein expression in GC tissues. Tissue sections were incubated with primary antibodies at $4{ }^{\circ} \mathrm{C}$ overnight. Goat-rabbit secondary antibodies were used to combine the primary antibodies. The protein location and expression intensity were visualized by
3,3-Diaminobenzidine tetrahydrochloride (DAB; Zhongshan Golden Bridge Biotechnology, Inc.). The staining results for the SOX4 were semi-quantitatively calculated by multiplying the staining intensity and the percentage of positive normal cells, as previously reported (13).

Luciferase reporter assay. The 3'-untranslated region (UTR) sequence of SOX4 predicted to interact with miR-381 and a mutation type were synthesized and inserted into the XbaI and FseI sites of the pGL3 control vector (Promega Corporation, Fitchburg, WI, USA). These constructs were named as wild-type SOX4 3'-UTR and mutant SOX4 3'-UTR. The reporter luciferase activity assay was performed using the Dual-Luciferase ${ }^{\circledR}$ Reporter Assay System (Promega Corporation) according to the instructions.

Western blot analysis. A total of $40 \mu \mathrm{g}$ protein of each sample isolated by RIPA lysis buffer (BioMed, China) was separated on SDS-PAGE gel and transferred onto a nitrocellulose membrane (Invitrogen). The membranes were incubated with the following primary rabbit anti-human antibodies purchased from Abcam Biotechnology, Inc. at $4^{\circ} \mathrm{C}$ overnight: SOX4 (ab80261, dilution at 1:1,000), E-cadherin (ab15148, dilution at 1:1,000), N-cadherin (ab18203, dilution at 1:1,000), Vimentin (ab16700, dilution at 1:1,000) and GAPDH (ab9485, dilution at 1:1,000). Then, the membranes were incubated with HRP-conjugated goat anti-rabbit secondary antibodies (ASR1038, ABGENT, WuXi, China, dilution at 1:5,000), and the signal were detected using the Bio-Rad Gel imaging system.

Statistical analysis. All quantitative data are expressed as (mean \pm SD). The Statistical Product and Service Solutions v21.0 software (SPSS 21.0, IBM, Armonk, New York, USA) was used to perform statistical analysis. The Pearson's Chi-square test and the Student's t-test were performed in this study. $\mathrm{P}<0.05$ showed statistically significant difference.

\section{Results}

MiR-381 expression is downregulatedted in GC tissues. We detected the expression of miR-381 by qRT-PCR in 60 paired GC tissues and tumor adjacent tissues. Compared with tumor adjacent tissues, lower expression level of miR-381 was detected in GC tissues $(\mathrm{P}<0.05$, Fig. 1A). Further, we divided these 60 GC patients into two groups according to their metastatic status. PCR results showed that the expression of miR-381 in tumors with metastasis was significantly lower than those without metastasis $(\mathrm{P}<0.05$, Fig. 1B). To determine the clinical values of miR-381, we used the mean expression level of miR-381 as a cut-off value to divide $60 \mathrm{GC}$ tissues into two groups: miR-381 high expression group $(n=24)$ and miR-381 low expression group $(n=36)$. As shown in Table I, low expression of miR-381 was associated with lymphatic metastasis $(\mathrm{P}=0.031)$ and advanced TNM stage (III+IV, $\mathrm{P}=0.003)$. These results indicated that miR-381 was related to tumor metastasis.

MiR-381 was associated with migration and invasion of SGC-7901 cells. Because the expression of miR-381 was related to the metastasis in gastric cancer, we wanted to confirm 
Table I. Clinical correlation of miR-381 expression in GC $(n=60)$.

\begin{tabular}{|c|c|c|c|c|}
\hline \multirow[b]{2}{*}{$\begin{array}{l}\text { Clinical } \\
\text { characteristics }\end{array}$} & \multicolumn{2}{|c|}{ miR-381 } & \multirow[b]{2}{*}{$\chi^{2}$} & \multirow[b]{2}{*}{ P-value } \\
\hline & $\begin{array}{c}\text { High } \\
\text { expression } \\
n=24\end{array}$ & $\begin{array}{c}\text { Low } \\
\text { expression } \\
n=36\end{array}$ & & \\
\hline Gender & & & 0.104 & 0.793 \\
\hline Male & 15 & 21 & & \\
\hline Female & 9 & 15 & & \\
\hline Age (year) & & & 0.100 & 0.797 \\
\hline$<50$ & 11 & 18 & & \\
\hline$\geq 50$ & 13 & 18 & & \\
\hline Hp infection & & & 3.665 & 0.068 \\
\hline Yes & 10 & 24 & & \\
\hline No & 14 & 12 & & \\
\hline $\begin{array}{l}\text { Lymphatic } \\
\text { metastasis }\end{array}$ & & & 5.602 & $0.031^{\mathrm{a}}$ \\
\hline Yes & 10 & 26 & & \\
\hline No & 14 & 10 & & \\
\hline $\begin{array}{l}\text { Histological } \\
\text { grade }\end{array}$ & & & 0.909 & 0.430 \\
\hline $\mathrm{I}+\mathrm{II}$ & 9 & 18 & & \\
\hline III+IV & 15 & 18 & & \\
\hline TNM stage & & & 9.074 & $0.003^{\mathrm{a}}$ \\
\hline $\mathrm{I}+\mathrm{II}$ & 20 & 16 & & \\
\hline III+IV & 4 & 20 & & \\
\hline
\end{tabular}

Hp, Helicobacter pylori; TNM, tumor-node-metastasis. ${ }^{\mathrm{P}}<0.05$.

whether miR-381 influenced migration and invasion on GC cells. First, we detected the expression levels of miR-381 in four GC cell lines (BGC-823, MGC-803, SGC-7901, MKN28) and a normal gastric epithelial cell line (GES-1). qRT-PCR data showed that the expression levels of miR-381 on these four GC cell lines, especially on SGC-7901 cells, were lower than that in GES-1 cells $(\mathrm{P}<0.05$, respectively, Fig. $2 \mathrm{~A})$. Next, we transfected miR-381 mimics into SGC-7901 cells to successfully upregulatedte its expression $(\mathrm{P}<0.001$, Fig. 2B). Then, we used wound healing assays to confirm that miR-381 over-expression significantly inhibited the migration of SGC-7901 cells $(\mathrm{P}<0.05$, Fig. 2C). Transwell assays also confirmed the effect of miR-381 for cell migration in vitro $(\mathrm{P}<0.05$, Fig. $2 \mathrm{D})$. Moreover, we still used the transwell assays to investigate the functions of miR-381 for cell invasion. As shown in Fig. 3E, compared with the negative control mimics group, upregulatedtion of miR-381 expression in SGC-7901 cells significantly decreased the invaded cell numbers $(\mathrm{P}<0.05)$. Taken together, these data suggested that miR-381 could attenuate the metastatic ability of SGC-7901 cells.

MiR-381 inhibited the epithelial-mesenchymal transition through downregulatedting SRY-Box 4. To investigate the mechanisms by which miR-381 inhibits GC metastasis, we
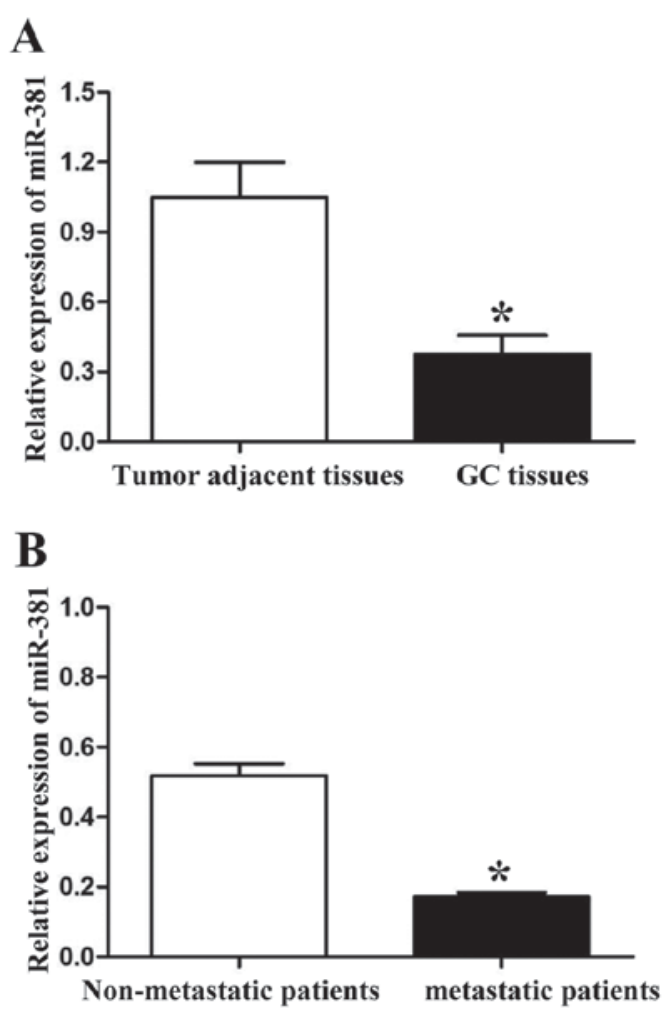

Figure 1. The expression levels of miR-381 in gastric carcinoma tissues. (A) The expression of miR-381 was downregulatedted in gastric carcinoma tissues. (B) Patients with metastasis got a lower miR-381 expression in the primary tumor tissues. ${ }^{*} \mathrm{P}<0.05$.

screened the target genes of miR-381 through online databases (TargetScan: http://www.targetscan.org/and MiRanda: http://www.microrna.org/). SOX4 was predicted as a potential downstream target of miR-381 according to the bioinformation analysis. To confirm the relationship between miR-381 and SOX4, first, we detect the expression of SOX4 in SGC-7901 cells which were transfected with miR-381 mimics. As shown in Fig. 3A, both the mRNA and protein expression levels were downregulatedted when we overexpressed miR-381 in SGC-7901 cells $(\mathrm{P}<0.05$, respectively). Second, we detected the protein expression levels of SOX4 in GC tissues by IHC and demonstrated that the protein expression of SOX4 was higher in miR-381 low-expression group than in miR-381 high-expression group ( $\mathrm{P}<0.05$, Fig. 3B). Finally, we used a Dual-Luciferase ${ }^{\circledR}$ Reporter Assay System to confirm that miR-381 decreased the luciferase activity of SOX4 containing a wild-type (wt) 3'-UTR but did not suppress the activity of SOX4 with a mutant (mt) 3'-UTR (P<0.01, Fig. 3C and 3D). Epithelial-mesenchymal transition (EMT) was characterized as an important mechanism for human cancer metastasis. Because Sox 4 has been considered as a master regulator of EMT in human cancers, we hypothesized that miR-381 could attenuate EMT of SGC-7901 cells through inhibiting SOX4 expression. So, we detected the protein expression levels of epithelial marker (E-cadherin) and mesenchymal markers (N-cadherin and vimentin) by immunoblotting. As shown in Fig. 3E, upregulatedtion of miR-381 in SGC-7901 cells resulted in significantly increase of E-cadherin expression $(\mathrm{P}<0.05)$ and decrease of $\mathrm{N}$-cadherin and vimentin expression $(\mathrm{P}<0.05$, respectively). 
A
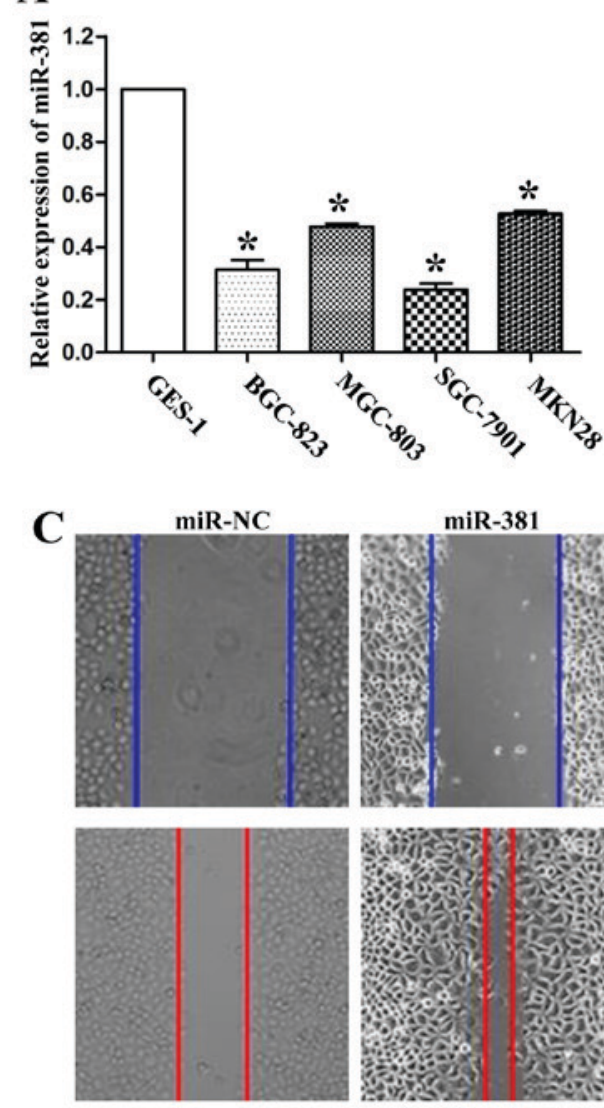

D
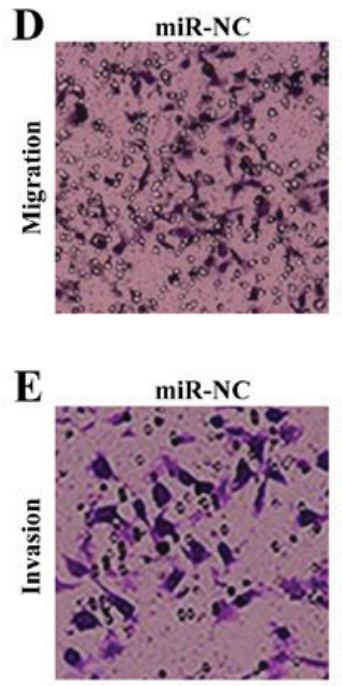

miR-381

miR-381
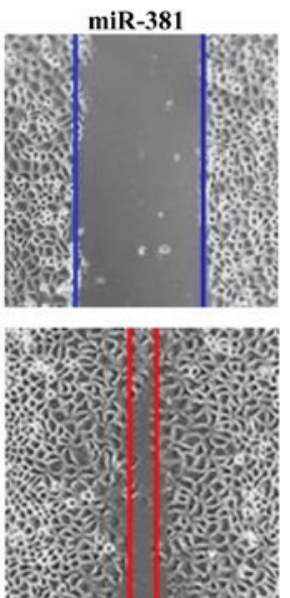

B
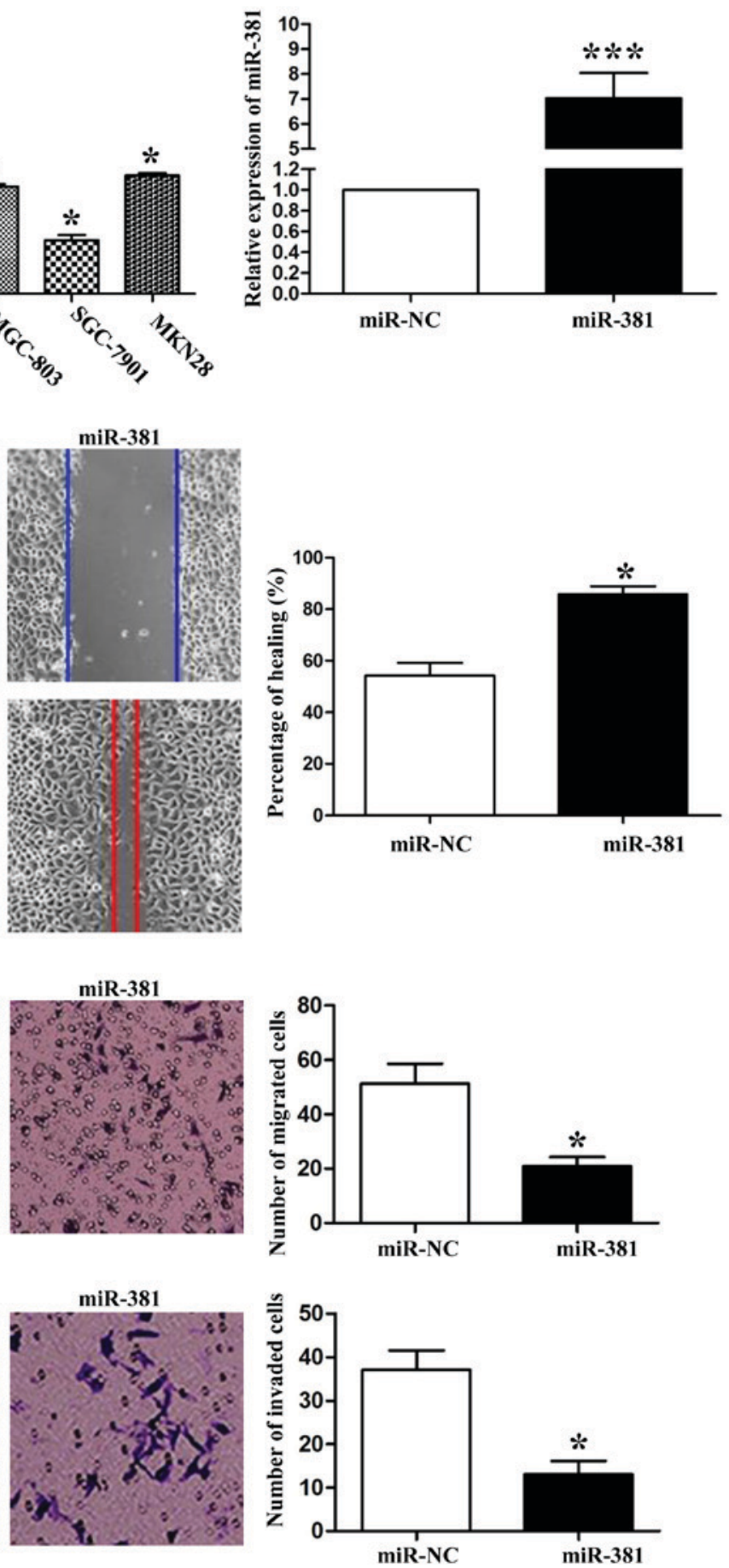

Figure 2. MiR-381 inhibited the migration and invasion of SGC-7901 cells. (A) The different relative expression levels of miR-381 in normal gastric epithelial cells and gastric carcinoma cells. (B) miR-381 mimics transfection upregulatedted the miR-381 expression in SGC-7901 cells. (C) Inhibition of miR-381 significantly reduced the migration speed of SGC-7901 cells. (D) Inhibition of miR-381 significantly decreased the migrated cell number. (E) Inhibition of miR-381 significantly decreased the invaded cell number. (A-E) Tests were repeated in triplicate with similar results. ${ }^{*} \mathrm{P}<0.05 ;{ }^{* * * *} \mathrm{P}<0.001$.

MiR-381 was negatively regulated by long noncoding RNA TUG1. It has been demonstrated that miRNAs can be downregulatedted through complementing with long noncoding RNAs (lncRNAs) (12). In this study, we found a negative relationship between long noncoding RNA TUG1 (IncRNA-TUG1) and miR-381 in GC tissues ( $\mathrm{P}<0.01$, Fig. 4A). LncRNA-TUG1 knockdown by siRNA could significantly increase miR-381 expression in SGC-7901 cells ( $\mathrm{P}<0.001$, Fig. 4B). These results partly explained the reason of low miR-381 expression in GC tissues.

\section{Discussion}

Metastasis including lymphatic metastasis and distant metastasis are the important risk factors for the poor prognosis of gastric carcinoma patients (7). Recently, microRNAs were 

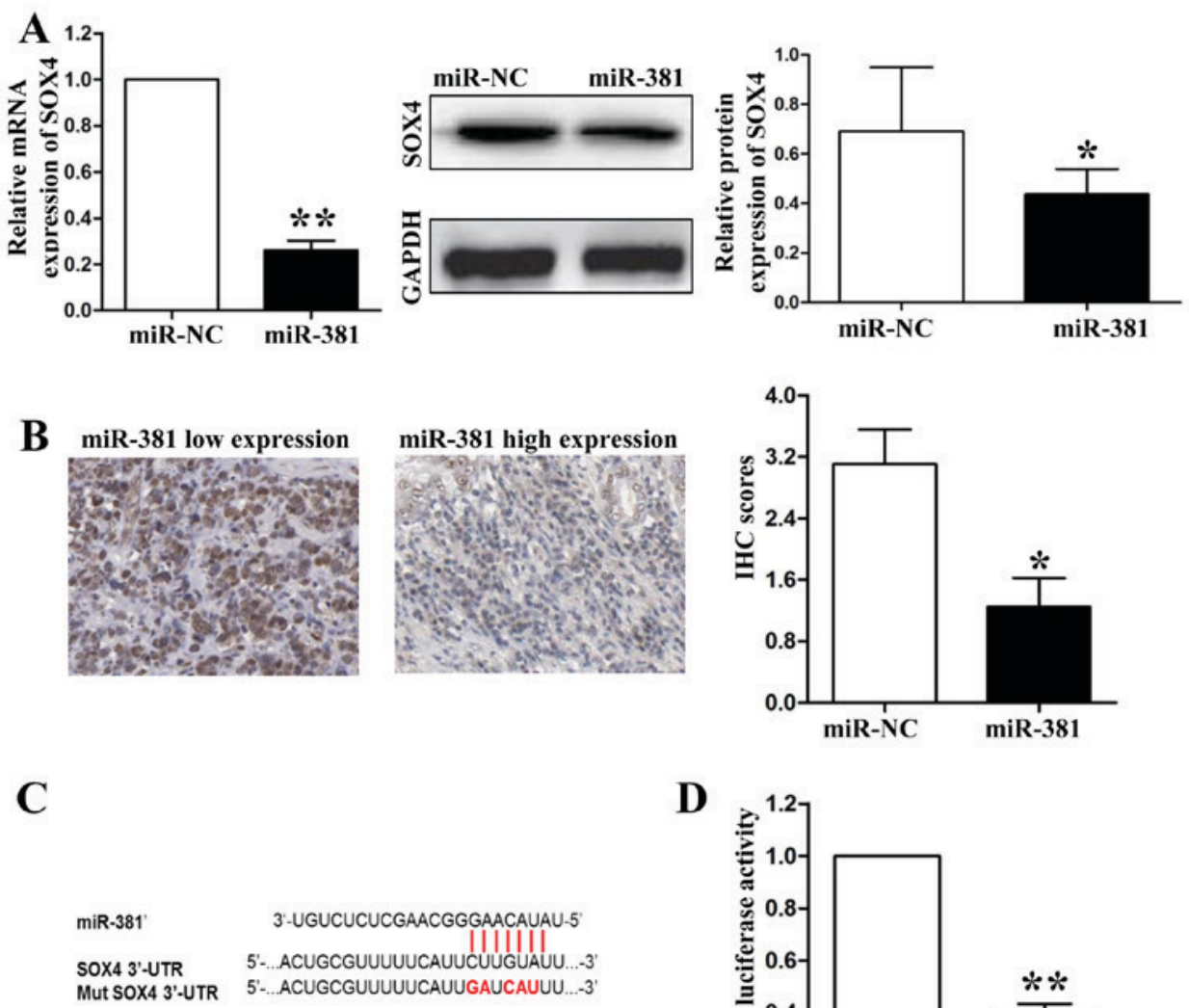

D
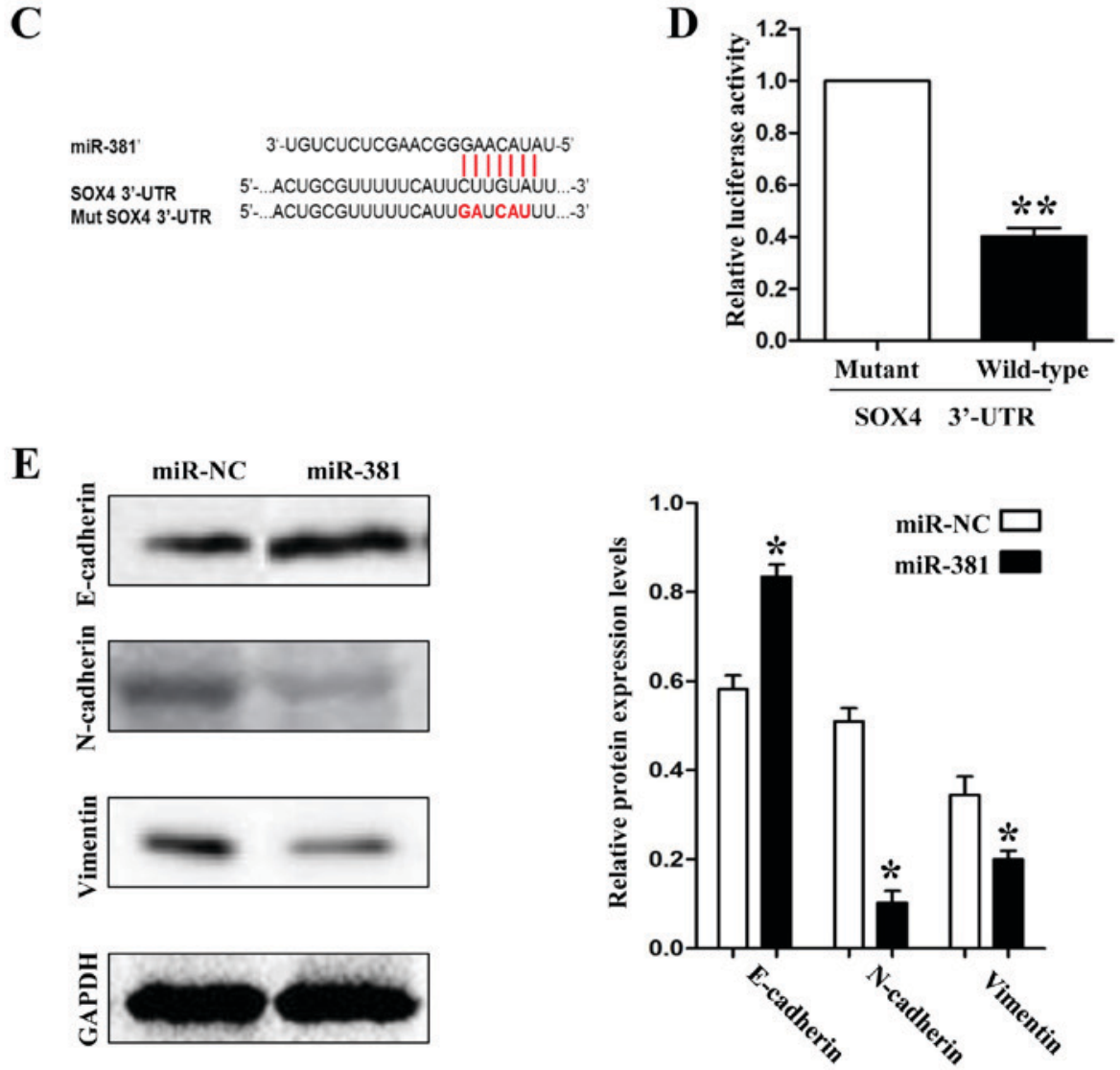

Figure 3. MiR-381 inhibited the EMT of SGC-7901 cells through targeting SOX4. (A) miR-381 overexpression significantly decreased SOX4 mRNA and protein expression. (B) Patients in miR-381 low expression group got high SOX4 protein expression levels. (C) The wild type and mutant type commentary sequences of SOX4 3'-UTR for miR-381. (D) miR-381 only decreased the luciferase activity that carried wild type but not mutant type 3'-UTR of SOX4. (E) Overexpression of miR-381 significantly increased E-cadherin expression while decreased N-cadherin and vimentin expression in SGC-7901 cells. (A-E) Tests were repeated in triplicate with similar results. ${ }^{*} \mathrm{P}<0.05 ;{ }^{* *} \mathrm{P}<0.01$.

demonstrated to play an important role in cancer metastasis (14). In breast cancer, miR-181b could inhibit CCL-18 induced cell migration and invasion through targeting NF- $\mathrm{KB}(15)$. In endometrial cancer, over-expression of microRNA-205 predicted tumor lymph node metastasis and poor prognosis (16). These evidences indicated that microRNAs might become a potential target for tumor metastasis control.
In the present study, we focused on miR-381, which exerted anti-cancer roles in breast cancer, osteosarcoma, colorectal cancer and hepatocellular carcinoma. However, the expression and biological functions in gastric carcinoma are still unclear. In order to elucidate these questions, first, we detected the expression levels of miR-381 in 60 paired gastric carcinoma tissues and matched tumor adjacent tissues, and found that 

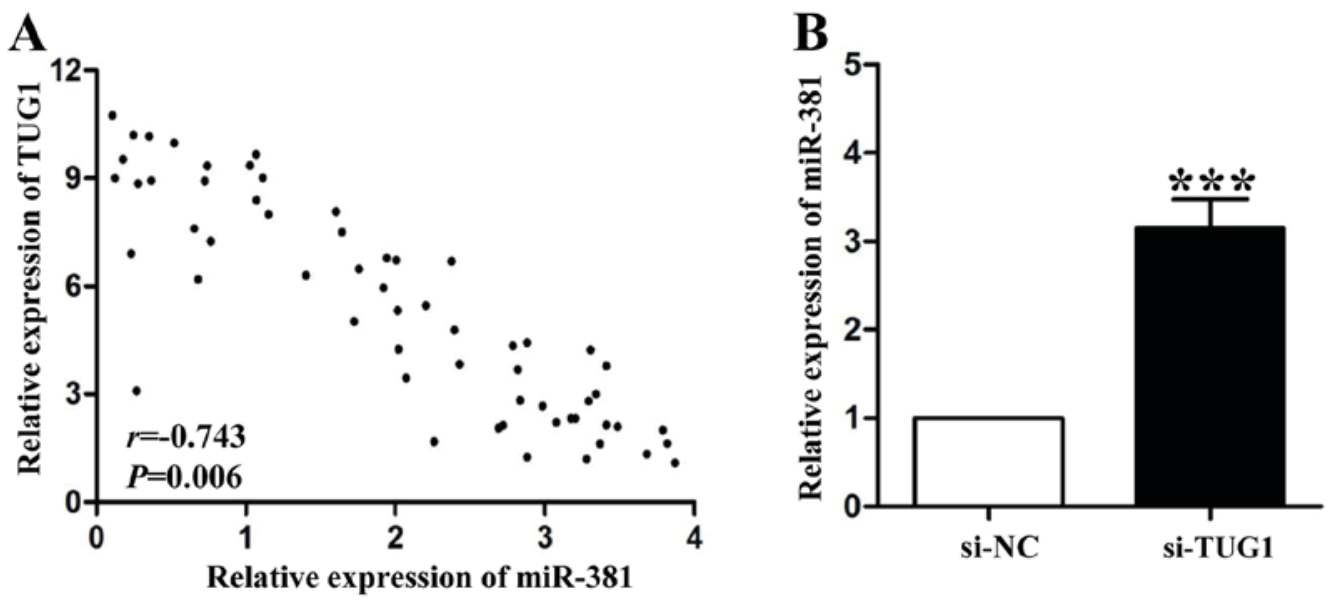

Figure 4. Long noncoding RNA TUG1 inhibited the expression of miR-381 in SGC-7901 cells. (A) Negative correlation between lncRNA-TUG1 and miR-381 is detected by real-time PCR for gene expression in 60 gastric carcinoma tissues $(r=-0.743, \mathrm{P}=0.006)$. (B) Inhibition of lncRNA-TUG1 by siRNA significantly reversed the expression of miR-381 in SGC-7901 cells. (A and B) Tests were repeated in triplicate with similar results. ${ }^{* * *} \mathrm{P}<0.001$.

miR-381 was downregulatedted in cancer tissues. Clinical data showed that low expression of miR-381 are correlated to lymphatic metastasis and advanced TNM stage. Interestingly, we demonstrated that gastric carcinoma tissues with metastasis had lower expression levels of miR-381 than those without metastasis. These results indicated that miR-381 could be served as a metastasis suppressor in gastric carcinoma.

Previous studies have shown that miR-381 has various biological functions in human cancers. For tumor growth, miR-381 could inhibit cell proliferation in colon cancer through targeting LRH-1 (17), in glioma through targeting BRD7 (17) and in renal cancer through targeting WEE1 (18). MiR-381 also could sensitize glioblastoma cells to temozolomide by targeting neurofilament light polypeptide (19). In the present study, the results of wound healing assays showed that over-expression of miR-381 inhibited the migration of gastric carcinoma cells. The transwell chamber assays further confirmed this effect. Moreover, the transwell results also showed that upregulatedtion of miR-381 suppressed cell invasion remarkably. Therefore, our results showed that miR-381 could impair the metastatic ability of gastric carcinoma cells.

Online databases result showed that SOX4, a member of the SOX (SRY-related HMG-box) family of transcription factors, might be a direct target of miR-381. In vitro, we demonstrated that miR-381 inhibited SOX4 expression through binding to its 3'-UTR in SGC-7901 cells. Moreover, patients with low expression levels of miR-381 exerted high SOX4 protein expression levels. EMT caused tumor metastasis has been well established (20). Even, in general, SOX4 didn't bind to the promoters of EMT markers to regulate their expression (21), but SOX4 has been demonstrated to promote the EMT in lung cancer (22) and prostate cancer (23). Data showed that a SOX4/EZH2 protein complex could be formed to bind to promote serval EMT related transcription factors expression, such as Snail, Zeb, and Twist (20). In order to know whether miR-381/SOX4 axis could regulate the EMT in gastric cancer, we detected three markers of EMT and found that miR-381 overexpression increased the endothelial marker's (E-cadherin) expression whereas inhibited mesenchymal makers' (N-cadherin and vimentin) expression. These results may explain why miR-381 could inhibit migration and invasion of SGC-7901 cells.

LncRNA TUG1 has been demonstrated to be overexpressed in gastric carcinoma and acts a metastasis inducer (24). In the present study, we confirmed a negative correlation between lncRNA TUG1 and miR-381 in their expression levels. The expression of miR-381 can be rescued after inhibition of lncRNA TUG1 in vitro. These demonstrated that lncRNA TUG1 could inhibit the expression of miR-381 in gastric cancer cells.

In conclusion, the present study demonstrates for the first time that the expression of miR-381 is significantly decreased in gastric cancer tissues, especially in cancer tissues with metastasis. MiR-381 can inhibit the metastatic behaviors and EMT of SGC-7901 cells. Furthermore, IncRNA TUG1 can inhibit the expression of miR-381 in gastric carcinoma.

\section{References}

1. Zeng YJ, Zhang CD and Dai DQ: Impact of lymph node micrometastasis on gastric carcinoma prognosis: A meta-analysis. World J Gastroenterol 21: 1628-1635, 2015.

2. Vilaseca Cabo A, Musquera Felip M, Ribal Caparros MJ and Alcaraz Asensio A: Metastasis of gastric carcinoma simulating a urothelial tumor. Case report and review of the literature. Arch Esp Urol 66: 885-889, 2013 (In English, Spanish).

3. Micolucci L, Akhtar MM, Olivieri F, Rippo MR and Procopio AD: Diagnostic value of microRNAs in asbestos exposure and malignant mesothelioma: Systematic review and qualitative meta-analysis. Oncotarget 7: 58606-58637, 2016.

4. Jiang L, Huang J, Chen Y, Yang Y, Li R, Li Y, Chen X and Yang D: Identification of several circulating microRNAs from a genome-wide circulating microRNA expression profile as potential biomarkers for impaired glucose metabolism in polycystic ovarian syndrome. Endocrine 53: 280-290, 2016.

5. Hansen TF, Andersen CL, Nielsen BS, Spindler KL, Sørensen FB, Lindebjerg J, Brandslund I and Jakobsen A: Elevated microRNA-126 is associated with high vascular endothelial growth factor receptor 2 expression levels and high microvessel density in colorectal cancer. Oncol Lett 2: 1101-1106, 2011.

6. Liu C, Wang C, Wang J and Huang H: miR-1297 promotes cell proliferation by inhibiting RB1 in liver cancer. Oncol Lett 12: 5177-5182, 2016.

7. Mataki H, Enokida H, Chiyomaru T, Mizuno K, Matsushita R, Goto Y, Nishikawa R, Higashimoto I, Samukawa T, Nakagawa M, et al: Downregulation of the microRNA-1/133a cluster enhances cancer cell migration and invasion in lung-squamous cell carcinoma via regulation of Coronin1C. J Hum Genet 60: 53-61, 2015. 
8. Ming J, Zhou Y, Du J, Fan S, Pan B, Wang Y, Fan L and Jiang J: miR-381 suppresses C/EBP $\alpha$-dependent $\mathrm{Cx} 43$ expression in breast cancer cells. Biosci Rep 35: pii: e00266, 2015.

9. Tzeng HE, Chang AC, Tsai $\mathrm{CH}$, Wang SW and Tang $\mathrm{CH}$ : Basic fibroblast growth factor promotes VEGF-C-dependent lymphangiogenesis via inhibition of miR-381 in human chondrosarcoma cells. Oncotarget 7: 38566-38578, 2016.

10. Li Y, Zhao C, Yu Z, Chen J, She X, Li P, Liu C, Zhang Y, Feng J, $\mathrm{Fu} \mathrm{H}$, et al: Low expression of miR-381 is a favorite prognosis factor and enhances the chemosensitivity of osteosarcoma Oncotarget 7: 68585-68596, 2016.

11. Xia B, Li H, Yang S, Liu T and Lou G: MiR-381 inhibits epithelial ovarian cancer malignancy via YY1 suppression. Tumour Biol 37: 9157-9167, 2016

12. Bayoumi AS, Sayed A, Broskova Z, Teoh JP, Wilson J, Su H, Tang YL and Kim IM: Crosstalk between long noncoding RNAs and MicroRNAs in health and disease. Int J Mol Sci 17: 356, 2016.

13. Li C, Yang W, Zhang J, Zheng X, Yao Y, Tu K and Liu Q SREBP-1 has a prognostic role and contributes to invasion and metastasis in human hepatocellular carcinoma. Int J Mol Sci 15: 7124-7138, 2014.

14. Wei WJ, Shen CT, Song HJ, Qiu ZL and Luo QY: MicroRNAs as a potential tool in the differential diagnosis of thyroid cancer: A systematic review and meta-analysis. Clin Endocrinol (Oxf) 84: 127-133, 2016.

15. Wang L, Wang YX, Chen LP and Ji ML: Upregulation of microRNA-181b inhibits CCL18-induced breast cancer cell metastasis and invasion via the NF- $\mathrm{KB}$ signaling pathway. Oncol Lett 12: 4411-4418, 2016.

16. Ma YJ, Ha CF, Bai ZM, Li HN, Xiong Y and Jiang J: Overexpression of microRNA-205 predicts lymph node metastasis and indicates an unfavorable prognosis in endometrial cancer. Oncol Lett 12: 4403-4410, 2016.
17. Liang Y, Zhao Q, Fan L, Zhang Z, Tan B, Liu Y and Li Y: Downregulatedtion of MicroRNA-381 promotes cell proliferation and invasion in colon cancer through upregulatedtion of LRH-1. Biomed Pharmacother 75: 137-141, 2015.

18. Chen B, Duan L, Yin G, Tan J and Jiang X: miR-381, a novel intrinsic WEE1 inhibitor, sensitizes renal cancer cells to 5-FU by upregulatedtion of $\mathrm{Cdc} 2$ activities in 786-O. J Chemother 25 229-238, 2013

19. Wang Z, Yang J, Xu G, Wang W, Liu C, Yang H, Yu Z, Lei Q, Xiao L, Xiong J, et al: Targeting miR-381-NEFL axis sensitizes glioblastoma cells to temozolomide by regulating stemness factors and multidrug resistance factors. Oncotarget 6: 3147-3164, 2015.

20. Yeung KT and Yang J: Epithelial-mesenchymal transition in tumor metastasis. Mol Oncol 11: 28-39, 2017.

21. Parvani JG and Schiemann WP: Sox4, EMT programs, and the metastatic progression of breast cancers: Mastering the masters of EMT. Breast Cancer Res 15: R72, 2013

22. Li Y, Chen P,Zu L, Liu B, Wang M and Zhou Q: MicroRNA-338-3p suppresses metastasis of lung cancer cells by targeting the EMT regulator Sox4. Am J Cancer Res 6: 127-140, 2016.

23. Fu W, Tao T, Qi M, Wang L, Hu J, Li X, Xing N, Du R and Han B: MicroRNA-132/212 upregulation inhibits TGF- $\beta$-Mediated epithelial-mesenchymal transition of prostate cancer cells by targeting SOX4. Prostate 76: 1560-1570, 2016.

24. Ji TT, Huang X, Jin J, Pan SH and Zhuge XJ: Inhibition of long non-coding RNA TUG1 on gastric cancer cell transference and invasion through regulating and controlling the expression of miR-144/c-Met axis. Asian Pac J Trop Med 9: 508-512, 2016. 